\title{
A Wearable Wireless Energy Link for Thin-Film Batteries Charging
}

\section{Giuseppina Monti, Laura Corchia, Egidio De Benedetto, and Luciano Tarricone}

Department of Engineering for Innovation, University of Salento, Via Monteroni, 73100 Lecce, Italy

Correspondence should be addressed to Giuseppina Monti; giuseppina.monti@unisalento.it

Received 26 November 2015; Accepted 28 February 2016

Academic Editor: Jaume Anguera

Copyright ( 2016 Giuseppina Monti et al. This is an open access article distributed under the Creative Commons Attribution License, which permits unrestricted use, distribution, and reproduction in any medium, provided the original work is properly cited.

A wireless charger for low capacity thin-film batteries is presented. The proposed device consists of a nonradiative wireless resonant energy link and a power management unit. Experimental data referring to a prototype operating in the ISM band centered at $434 \mathrm{MHz}$ are presented and discussed. In more detail, in order to facilitate the integration into wearable accessories (such as handbags or suitcases), the prototype of the wireless energy link was implemented by exploiting a magnetic coupling between two planar resonators fabricated by using a conductive fabric on a layer of leather. From experimental data, it is demonstrated that, at $434 \mathrm{MHz}$, the RF-to-RF power transfer efficiency of the link is approximately $69.3 \%$. As for the performance of the system as a whole, when an RF power of $7.5 \mathrm{dBm}$ is provided at the input port, a total efficiency of about $29.7 \%$ is obtained. Finally, experiments performed for calculating the charging time for a low capacity thin-film battery demonstrated that, for RF input power higher than $6 \mathrm{dBm}$, the time necessary for recharging the battery is lower than 50 minutes.

\section{Introduction}

In recent years, wearable electronics have gained much research interest, with applications that range from healthcare monitoring $[1,2]$ to public safety $[3,4]$ and mobile computing [5-7]. The design and fabrication of wearable electronics require that the embedding of electronic components inside clothes and/or other wearable accessories must not compromise the appearance and usability of the product. In fact, in the fashion industry, any new wearable technology must be useful, but also comfortable. Additionally, it must not be intrusive to the user, who must be able to carry out his daily activities without any movement limitation and/or additional burden.

Considering these requirements, either near-field [8-13] or far-field [14-21] wireless technologies may successfully serve the purpose for both data and power transmission. Still, to guarantee a seamless integration of electronic devices and antennas in wearable and portable accessories, it is crucial to select appropriate materials and fabrication techniques. To this purpose, the use of nonconventional materials such as textile materials, conductive threads, electrotextile fabrics, and nonwoven conductive fabrics should be preferred.

Accordingly, some wearable antennas for far-field Wireless Power Transmission (WPT) links have been proposed in the literature [15-17]. Among these, in [16], numerical data referring to a multifrequency rectifying antenna (rectenna) are reported: the multiband behavior is obtained by using a slotted annular-ring microstrip antenna. The overall system is a multilayer structure using two layers of pile, the Global EMC shielding fabric for the conductive parts, a layer of Kapton for the rectifying circuit, and a thermoadhesive layer at each interface between pile and conductive fabric.

Additionally, in [17], two textile logo antennas fabricated by means of a self-adhesive nonwoven conductive fabric have been presented.

As for near-field WPT links for wearable applications, a system using two resonators on a layer of leather has been proposed in [22].

In this paper, starting from the prototype presented in [22], a wireless battery charger for application in clothing industry is presented. In more detail, the wireless resonant 


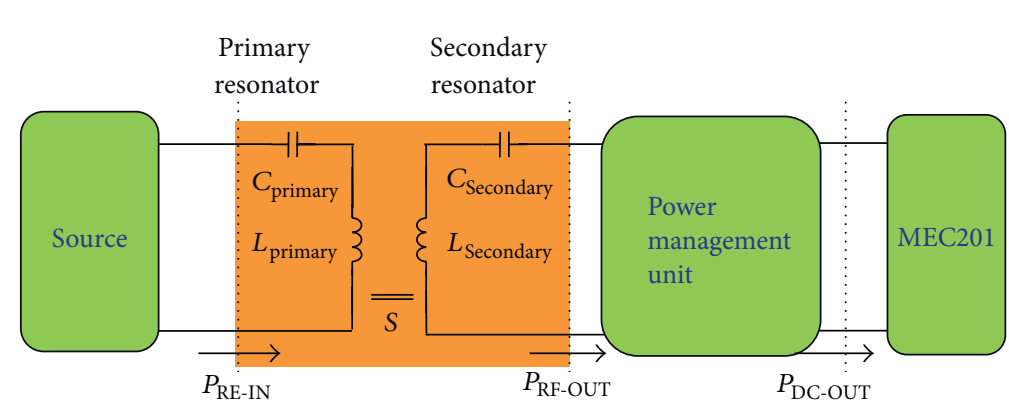

(a)

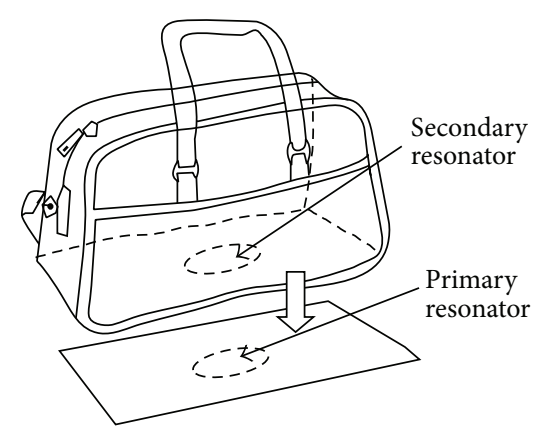

(b)

Figure 1: Proposed wireless charger. (a) Schematic representation: the link consists of a wireless resonant energy link and of a power management unit. (b) Example of application: the secondary resonator is embedded into a leather bag, while the primary resonator is on a pad where the bag should be placed on for charging the battery.

energy link (WREL) proposed in this work was exploited for implementing a wireless charger [8,9] for low capacity thin-film batteries. The proposed system consists of (1) two planar resonators that were optimized and fabricated to be embedded in portable leather accessories and (2) a power management unit (PMU).

Figure 1(a) shows a schematic representation of the proposed WPT link, which exploits a magnetic coupling between two planar resonators, namely, a primary resonator and a secondary resonator. The former is embedded into a pad and is connected to an external power source that provides it with an AC input power, whereas the latter is connected to the rechargeable battery by means of the PMU. Figure 1(b) shows a sketch of a possible practical application: the secondary resonator is embedded into a bag, while the primary resonator is embedded into a pad where the bag should be placed on for charging battery.

As will be detailed in the following section, for the resonators fabrication, a self-adhesive nonwoven fabric was used for the conductive parts, while a layer of leather was used as support.

Numerical and experimental results referring to a prototype working in the ISM band $(433.05-434.79 \mathrm{MHz})$ are reported and discussed.

The paper is structured as follows. Numerical and experimental data obtained for the proposed WPT link are illustrated in Section 2. Section 3 describes each single block of the PMU. Experimental results related to the proposed wireless charging system, as a whole, are reported in Section 4. Finally, conclusions are drawn in Section 5.

\section{Wearable Resonators Geometry and Results}

2.1. Resonators Geometry and Numerical Results. The proposed WPT link is comprised of two identical resonators, each one consisting of a distributed inductance loaded by a lumped capacitor [22]. Figure 2(a) shows a sketch of a perspective view of the configuration adopted for the WPT link, while Figure 2(b) illustrates the resonator geometry.

From Figure 2(a), it can be noticed that each resonator consists of an elliptical loop loaded by a smaller one. The two

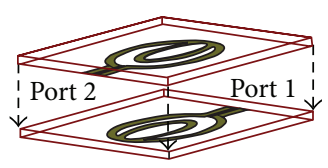

(a)

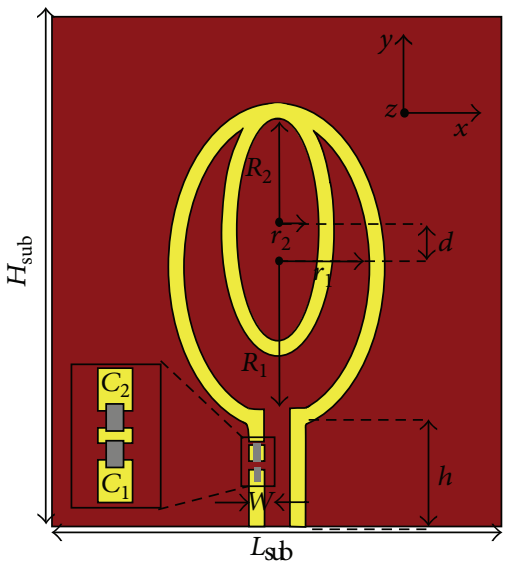

(b)

FIGURE 2: Geometry of the proposed wireless energy link: (a) perspective view; (b) front view. It can be seen that the secondary resonator is overlapped to the primary resonator.

resonators are rotated by $180^{\circ}$ with respect to each other (on the $x-y$ plane), and they are also aligned with the center of the external elliptical loop. Additionally, to tune the resonance frequency, two lumped capacitors, $C_{1}$ and $C_{2}$, are also used.

The geometry of the resonators was optimized, by means of full-wave simulations, to obtain (1) an operating frequency in the ISM band $(433.05-434.79 \mathrm{MHz})$ and (2) an input impedance of $50 \Omega$. In the following, the main steps of the design process are briefly described.

The starting geometry was a simple elliptical loop with a major axis of $36 \mathrm{~mm}$; a width of $2 \mathrm{~mm}$; and an eccentricity close to zero, thus corresponding to a nearly circular geometry. These initial values were chosen in order to obtain a compact structure, suitable to be fabricated with the facilities 


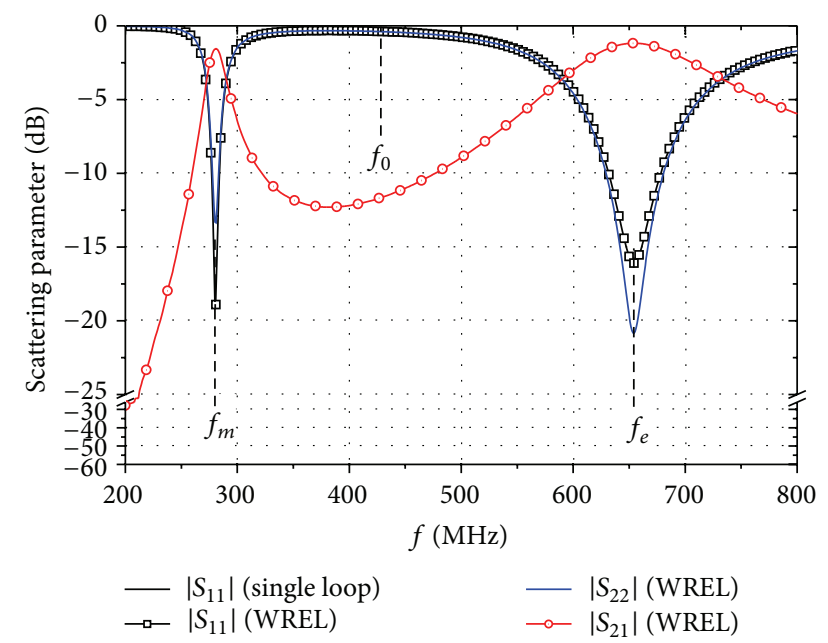

FIgURE 3: Simulated reflection coefficient of the single loop compared to the scattering parameters of the WREL consisting of two loops configured as in Figure 2(a).

at hand. To tune the resonance frequency in the ISM band, a series $1.1 \mathrm{pF}$ lumped capacitor was added.

The scattering parameters corresponding to this initial geometry are illustrated in Figure 3, which shows (1) the reflection coefficient of the primary resonator and (2) the scattering parameters of the wireless resonant energy link, in the configuration of Figure 2(a).

It can be seen that the resonance frequency, $f_{0}$, of the single resonator is approximately $427 \mathrm{MHz}$. It can be also noticed that when the two resonators are coupled, the resonance frequency $f_{0}$ splits into two different frequencies (i.e., the magnetic resonance $f_{m}$ and the electric resonance $f_{e}$ ) which correspond to two relative maxima of the power transfer efficiency. From the coupled mode theory, it can be derived that these frequencies are given by [23]

$$
\begin{gathered}
f_{m}=\frac{f_{0}}{(\sqrt{1+k})}, \\
f_{e}=\frac{f_{0}}{(\sqrt{1-k})},
\end{gathered}
$$

where $k$ is the magnetic coupling between the two resonators. In practical applications, $k$ assumes small values, and the two resonance frequency values are so mutually close that by acting on the resonators parameters a bandwidth centered at $f_{0}$ can be obtained where the link can be used for WPT applications.

Starting from these results, the geometry of the resonators was optimized in order to obtain a bandwidth suitable for WPT applications around $f_{0}$ (i.e., flat transmission scattering parameters in the frequency range between $f_{m}$ and $f_{e}$ ) and in order to center $f_{e}$ at the center of the ISM band of interest. The first step of the design process was the optimization of the eccentricity $(e)$ of the ellipse. In this regard, some full-wave simulation results are illustrated in Figure 4(a); in
TABLE 1: Dimensions in millimeters of the geometry illustrated in Figure 2.

\begin{tabular}{ccccccccc}
\hline$H_{\text {sub }}$ & $L_{\text {sub }}$ & $W$ & $R_{1}$ & $r_{1}$ & $R_{2}$ & $r_{2}$ & $h$ & $d$ \\
\hline 54.7 & 47.6 & 2.4 & 15.2 & 9.5 & 11.3 & 3.7 & 11.9 & 3.9 \\
\hline
\end{tabular}

the frequency range of interest, the best results were obtained for an eccentricity of about 0.87 .

The design process was carried out by optimizing the width $(W)$ of the loop; the corresponding results are given in Figure 4(b). The parameter $W$ mainly influences the distributed equivalent inductance and capacitance of each loop and thus $f_{0}$. As a consequence, a variation of $W$ leads to a shift of the entire useful bandwidth of the link. According to full-wave simulation results, $W$ was set at $2 \mathrm{~mm}$.

Later on, in order to improve the flatness of $S_{21}$ parameter, the possibility of improving the matching at $f_{0}$ by loading the elliptical loop with LC distributed elements was investigated. Satisfactory results were obtained by loading the external loop with a smaller loop. In Figure 5, the scattering parameters obtained for the final geometry are compared with the ones obtained for the simple elliptical resonators. As shown in Figure 5(b), the transmission coefficient (i.e., $S_{21}$ parameter) is higher than $-3 \mathrm{~dB}$ in the $263-582 \mathrm{MHz}$ frequency range, corresponding to a relative bandwidth of approximately $75.5 \%$. The geometric parameters of the final geometry, simultaneously optimized by means of full-wave simulations, are summarized in Table 1 . After optimization, the values of the lumped capacitors $\left(C_{1}\right.$ and $\left.C_{2}\right)$ are $6.8 \mathrm{pF}$ and $10 \mathrm{pF}$, respectively.

2.2. Experimental Results. A prototype of the proposed WPT link was fabricated by using an adhesive nonwoven conductive fabric [24] on a layer of leather. Figure 6(a) shows a picture of the two realized resonators, while Figure 6(b) shows the setup adopted for experimental tests.

The conductive fabric has a surface resistivity of $0.04 \Omega /$ sq. With reference to the fabrication of devices for wearable applications, this fabric has several advantages, such as low cost, mechanical resistance, and no fraying problems. This last feature makes it particularly suitable also for the fabrication of complicated geometries [15, 17, 22, 25, 26].

As for the leather substrate, a layer with a thickness of $1.65 \mathrm{~mm}$ and a relative dielectric permittivity $\left(\varepsilon_{r}\right)$ of 3 was used. The value of $\varepsilon_{r}$ was determined by fitting numerical and experimental results obtained for some simple rectangular patches. As for the lumped capacitors, $C_{1}$ and $C_{2}$, surface mounted capacitors were used.

The $S$-parameters were measured through a vector network analyzer R\&S ZVA50: the obtained results are reported in Figure 7. An overall good agreement can be noticed between the measurement results and the numerical data illustrated in Figure 5. In more detail, the measured transmission coefficient is higher than $-3 \mathrm{~dB}$ in the frequency range $319-524 \mathrm{MHz}$, corresponding to a relative bandwidth of about $48.6 \%$. The slight degradation of the measured performance with respect to the simulated one is most likely 


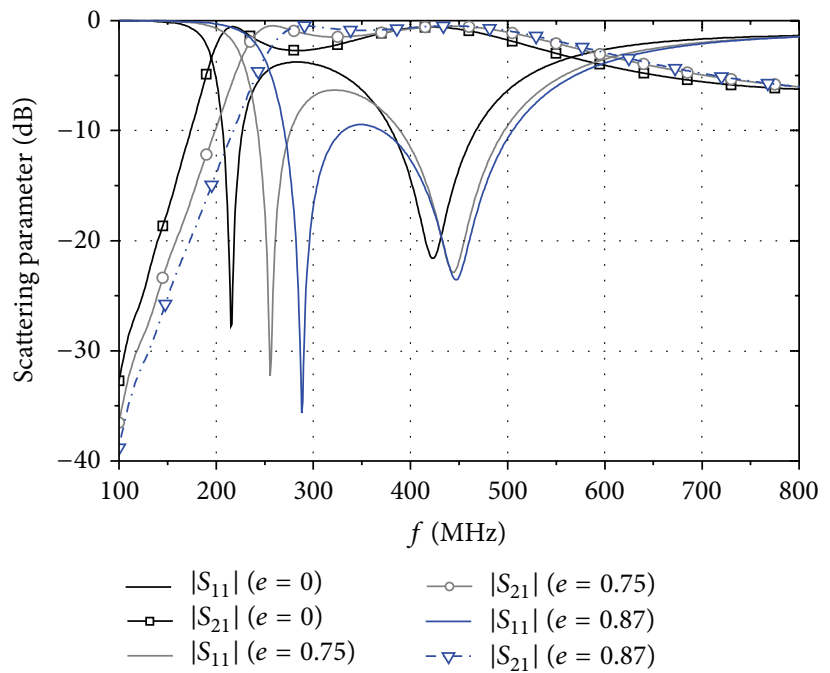

(a)

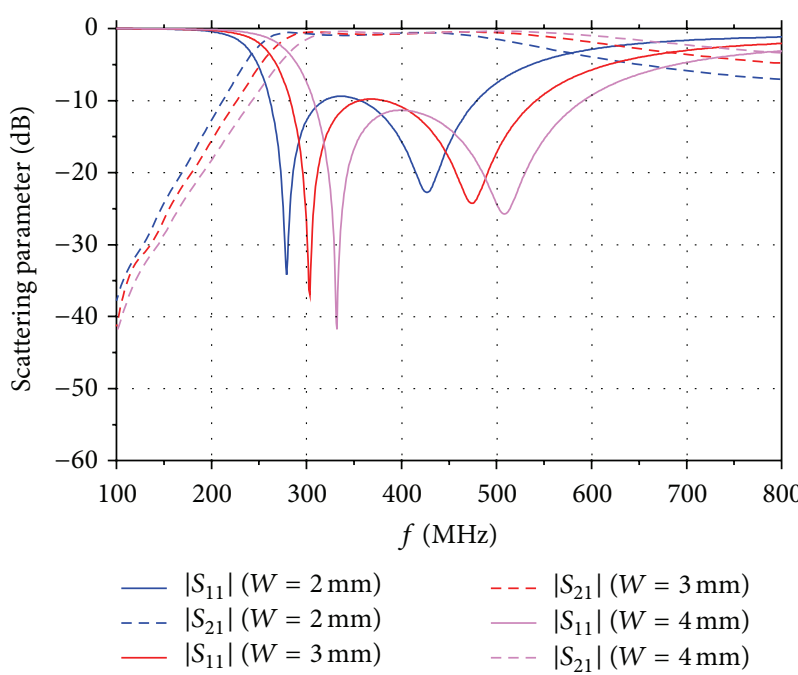

(b)

FIGURE 4: Scattering parameters calculated by means of full-wave simulations during the optimization process of the resonators. Optimization of (a) the eccentricity $e$ and (b) the width, $W$, of the loop.

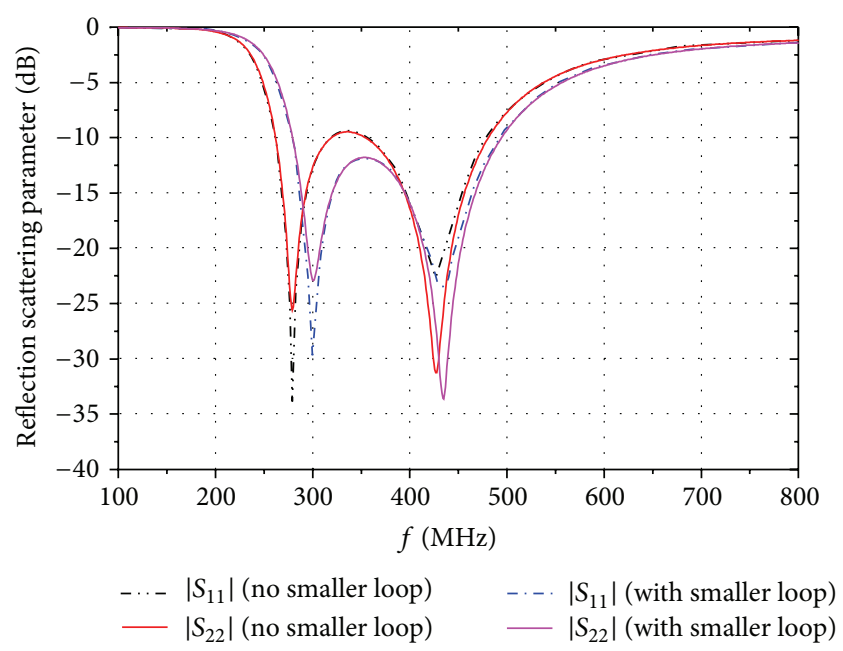

(a)

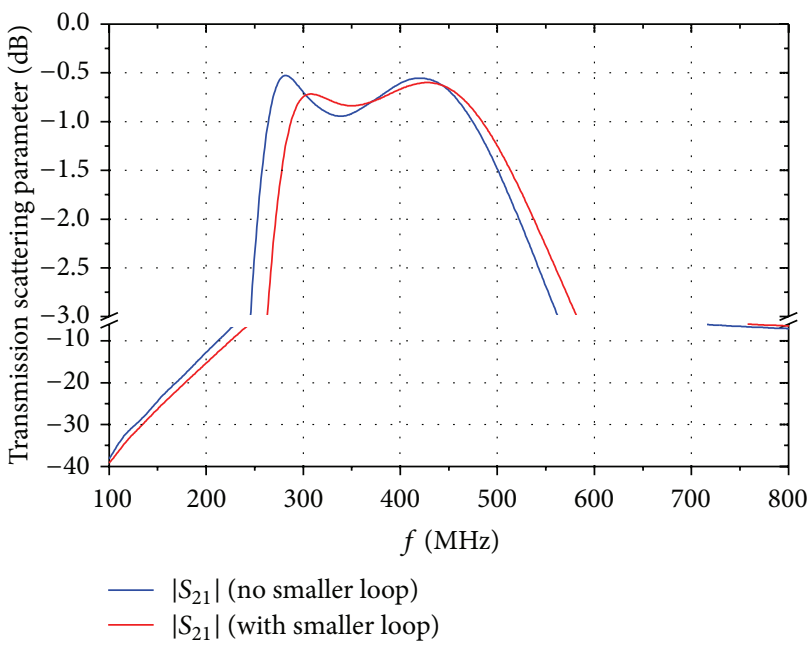

(b)

FIGURE 5: Comparison between the scattering parameters, calculated by means of full-wave simulations, for the proposed wireless energy link, obtained with and without the smaller loop: (a) reflection scattering parameters; (b) transmission scattering parameters.

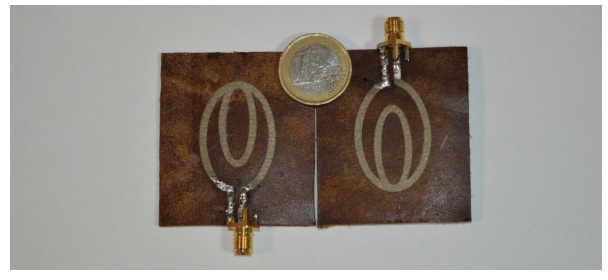

(a)

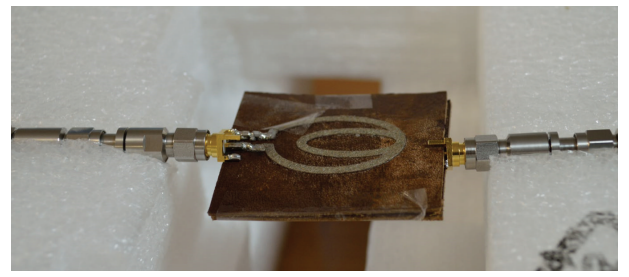

(b)

FIGURE 6: Picture of the fabricated prototype: (a) the two resonators; (b) measurements setup. 


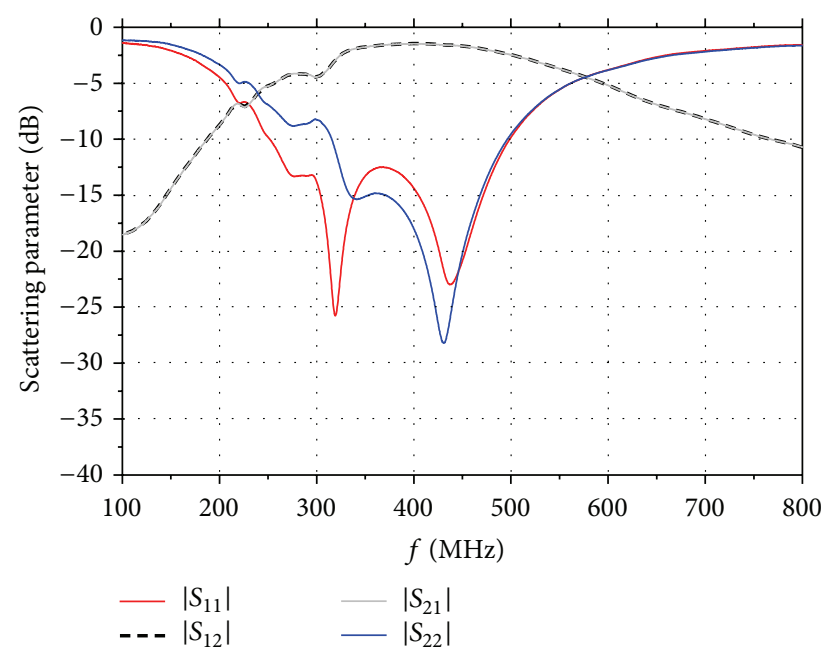

FIGURE 7: $S$-parameters measured by using the experimental configuration shown in Figure 6(b).

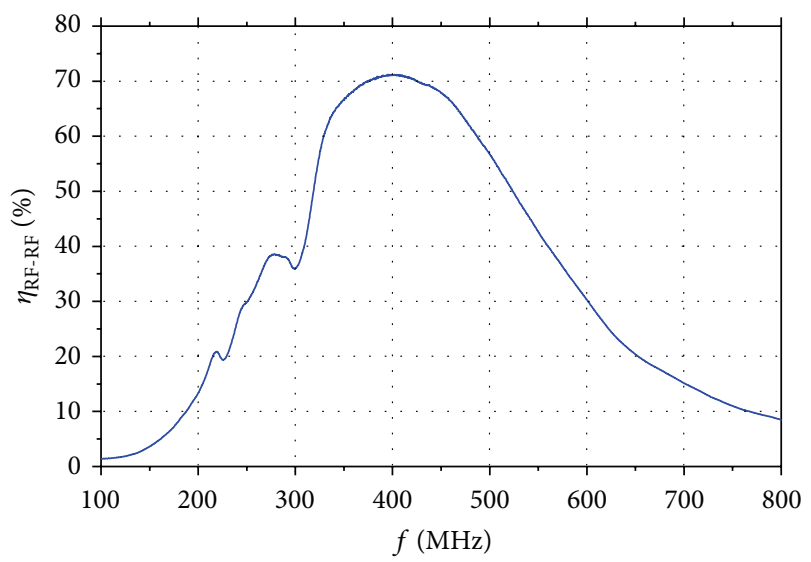

FIGURE 8: Experimental results obtained for the power transfer efficiency $\left(\eta_{\mathrm{RF}-\mathrm{RF}}\right)$ of the proposed wireless energy link.

due to the SMA connectors, which do not allow a perfect overlap between the two resonators.

With reference to the schematization shown in Figure 1, by denoting by $P_{\mathrm{RF}-\mathrm{IN}}$ the power available from the source and by $P_{\text {RF-OUT }}$ the power delivered by the secondary resonator to the PMU unit, the RF-to-RF power transfer efficiency $\left(\eta_{\mathrm{RF}-\mathrm{RF}}\right)$ can be defined as follows:

$$
\eta_{\mathrm{RF}-\mathrm{RF}}=\frac{P_{\mathrm{RF}-\mathrm{OUT}}}{P_{\mathrm{RF}-\mathrm{IN}}}=\frac{P_{\mathrm{RF}-\mathrm{IN}} \cdot\left|S_{21}\right|^{2}}{P_{\mathrm{RF}-\mathrm{IN}}}=\left|S_{21}\right|^{2} .
$$

Figure 8 shows the power transfer efficiency calculated for the proposed WPT link by using experimental data according to (2). A maximum of $71.3 \%$ was obtained at $403.97 \mathrm{MHz}$. It can also be noticed that $\eta_{\mathrm{RF}-\mathrm{RF}}$ is higher than $50 \%$ in the frequency range $318.70-523.67 \mathrm{MHz}$ corresponding to a relative bandwidth of approximately $48.7 \%$. Furthermore, it can be seen that, in the ISM band $(433.05-434.79 \mathrm{MHz})$, the proposed link exhibits values of $\eta_{\text {RF-RF }}$ higher than $69.3 \%$.

\section{Power Management Unit}

To assess the feasibility of using the proposed WPT link for implementing a wireless charger, a PMU that converts the RF power received by the secondary resonator into a DC power that can be directly delivered to a battery was also designed. As can be seen from the schematic representation given in Figure 9, the PMU consists of three major blocks: (A) a voltage doubler rectifier, (B) a boost circuit, and (C) a battery charger. A photograph of a prototype fabricated on a double side FR4 board (relative dielectric permittivity equal to 4.1 and thickness of $0.4 \mathrm{~mm}$ ) is shown in Figure 10. The size of the PMU board is $55 \times 55 \mathrm{~mm}^{2}$.

In the following paragraphs, each single block will be described in detail.

3.1. Voltage Doubler Rectifier. A voltage doubler rectifier was used as RF-to-DC rectifier; the corresponding schematic representation is reported in Figure 11. The design of the rectifier was developed by means of the software NI AWR Design Environment by National Instruments [27]; the design goals were (1) to maximize the RF-to-DC conversion efficiency $\left(\eta_{\text {RF-DC }}\right)$ and (2) to obtain an input impedance of $50 \Omega$.

First of all, experimental tests were performed in order to determine the input impedance of the boost converter which is the load impedance of the rectifier (i.e., $Z_{\text {LOAD }}$ ). This way, a value of $Z_{\mathrm{LOAD}}$ of about $20 \mathrm{k} \Omega$ was derived. As for the value assumed for $P_{\text {RF-OUT }}$, which is the power delivered by the secondary resonator to the rectifier, a value of $5 \mathrm{dBm}$ at $434 \mathrm{MHz}$ was assumed. Considering that, from experimental results, the RF-to-RF efficiency of the WREL link presented in the previous section is equal to $69.3 \%$, assuming a value of $5 \mathrm{dBm}$ for $P_{\text {RF-OUT }}$ corresponds to assuming a value of about $6.5 \mathrm{dBm}$ for the power $P_{\mathrm{RF}-\mathrm{IN}}$ delivered to the primary resonator (see Figure 1), which is a reasonable value for WPT applications.

As can be seen from Figure 11, an Input Matching Network (IMN) consisting of two shunt varactors and a series inductor was used to match the rectifier to $50 \Omega$. The varactors $C_{\mathrm{VAR} 1}$ and $C_{\mathrm{VAR} 2}$ have a capacitance range of $0.65-2.5 \mathrm{pF}$ and of $2.5-6.5 \mathrm{pF}$, respectively. They were exploited during experimental tests to adjust the level of matching between the wireless energy link and the rectifier. As for the inductor, its value is $39 \mathrm{nH}$. The Schottky diodes are the HSMS-2820 by Avago Technologies [28]. The capacitors $C_{3}$ and $C_{4}$ of the voltage doubler rectifier were set at $0.1 \mu \mathrm{F}$.

The performance of the voltage doubler rectifier, in terms of $\eta_{\text {RF-DC }}$, was experimentally evaluated. Measurements were performed for different values of the load $\left(Z_{\mathrm{LOAD}}\right)$ and of the input power at the rectifier $\left(P_{\mathrm{RF}-\mathrm{OUT}}\right)$. The following definition was used to calculate $\eta_{\mathrm{RF}-\mathrm{DC}}$ values:

$$
\eta_{\text {RF-DC }}=\frac{P_{\text {Rect-OUT }}}{P_{\text {RF-OUT }}}=\frac{\left(V_{\text {Rect-OUT }}^{2} / Z_{\text {LOAD }}\right)}{P_{\text {RF-OUT }}},
$$

where $V_{\text {Rect-OUT }}$ is the DC voltage at the output port of the rectifier (see Figure 11). Experimental tests were performed by using the Vector Signal Generator (VSG) R\&S FSW26 to generate the RF signal at the input port of the rectifier (i.e., 
(a) Boost circuit

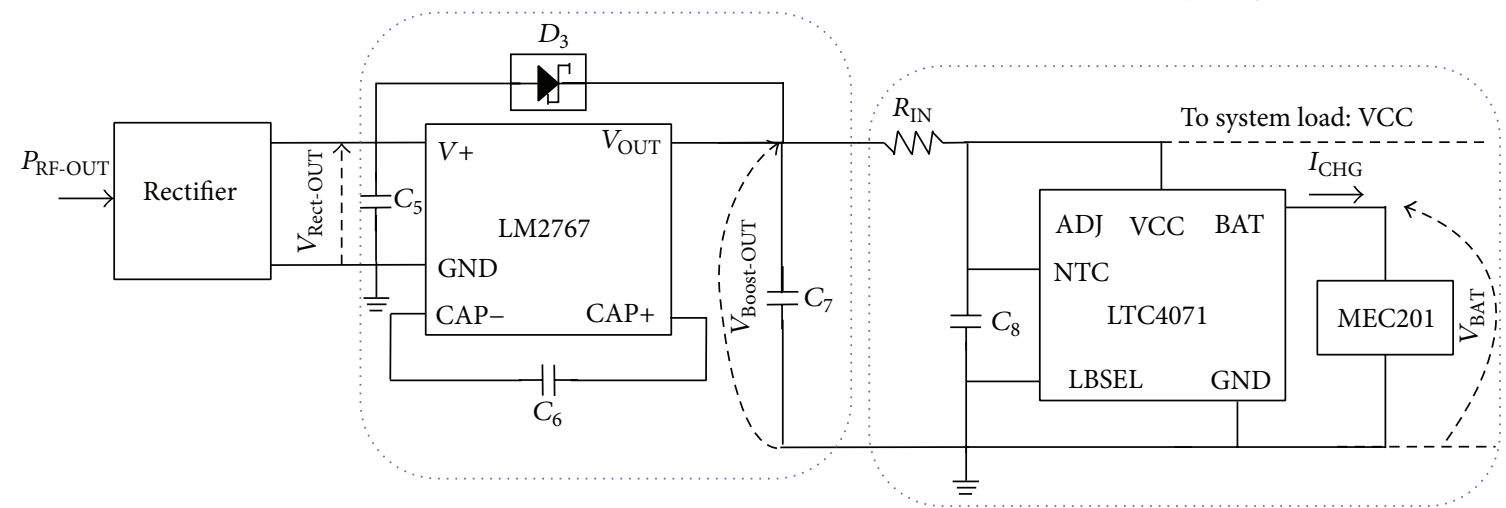

FIGURE 9: Schematic representation of the power management unit (see Figure 1(a)). In particular, the external lumped elements and the pins connection used for implementing the boost circuit (a) and the battery charger (b) are illustrated.

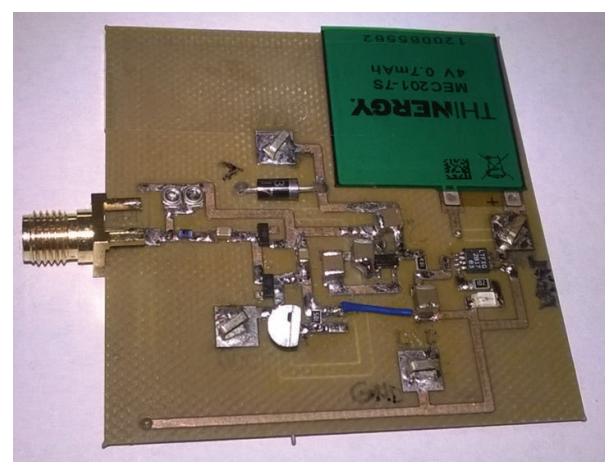

FIgURE 10: Prototype of the proposed power management circuit.

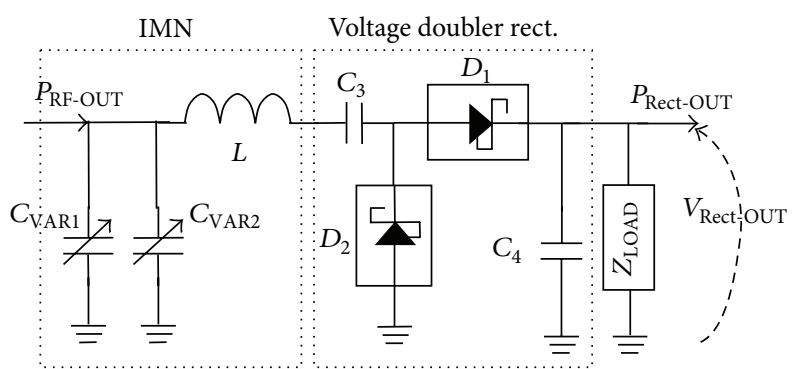

FIGURE 11: Schematic representation of the voltage doubler rectifier used for rectifying the RF signal.

$\left.P_{\text {RF-OUT }}\right)$. Results are reported in Figure 12, which shows the measured $\eta_{\text {RF-DC }}$ values as a function of the resistive load $(R)$, for different values of $P_{\text {RF-OUT }}$. It can be seen that a maximum of $54 \%$ was obtained for $R=3.3 \mathrm{k} \Omega$ and $P_{\mathrm{RF}-\mathrm{OUT}}=10 \mathrm{dBm}$. Furthermore, values of $\eta_{\text {RF-DC }}$ higher than $30 \%$ were obtained for $P_{\text {RF-OUT }}$ equal to $5 \mathrm{dBm}$ and values of $R$ in the range of 1$22 \mathrm{k} \Omega$.

3.2. Boost Circuit. The core of the boost circuit is the Texas Instruments LM2767 switched capacitor voltage converter [29]. This chip operates as a voltage doubler for an input

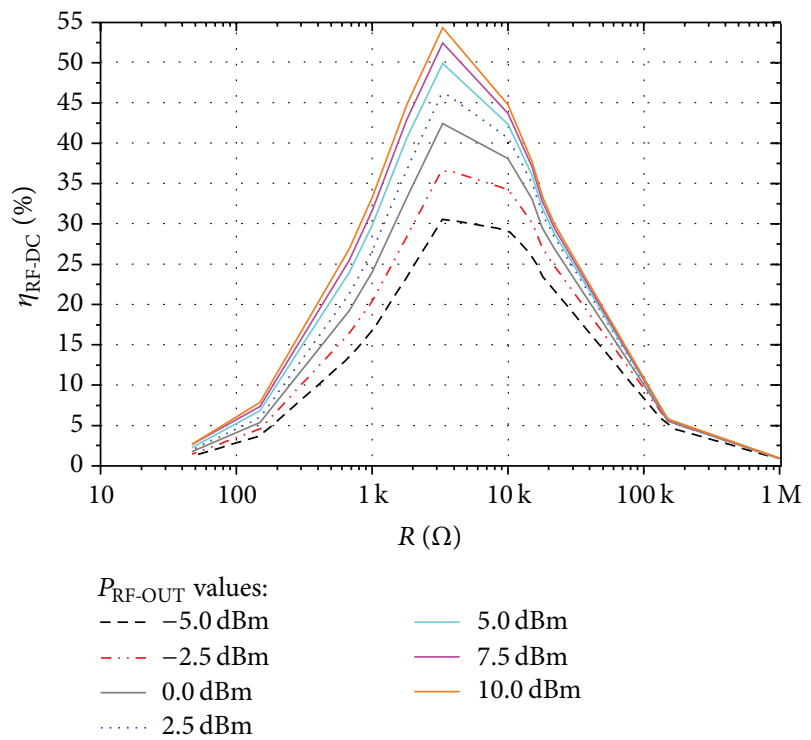

FIGURE 12: RF-to-DC conversion efficiency of the rectifier as function of the resistive load $(R)$, for different values of the RF power at the input port of the rectifier $\left(P_{\mathrm{RF}-\mathrm{OUT}}\right)$.

voltage in the range of $1.8 \mathrm{~V}$ to $5.5 \mathrm{~V}$ and a supply current of $40 \mu \mathrm{A}$. From specifications, the LM2767 guarantees an operating efficiency greater than $90 \%$ with most loads.

In Figure 9(a), a schematic representation of the circuital configuration adopted for implementing the boost converter is shown. The working mechanism is as follows: a DC voltage applied at the input pin (i.e., $V+$ ) is doubled at the output pin $\left(V_{\text {OUT }}\right)$. In the intended application, $V+$ is the voltage at the output port of the rectifier.

As recommended by the manufacturer, three lumped capacitors with low ESR (Equivalent Series Resistance) were used to maximize efficiency and reduce the output voltage drop and voltage ripple. In particular, three $10 \mu \mathrm{F}$ ceramic capacitors were employed for $C_{5}, C_{6}$, and $C_{7}$. The aim of the Schottky diode $D_{3}$ is to prevent malfunctions caused by possible internal latch-up. 


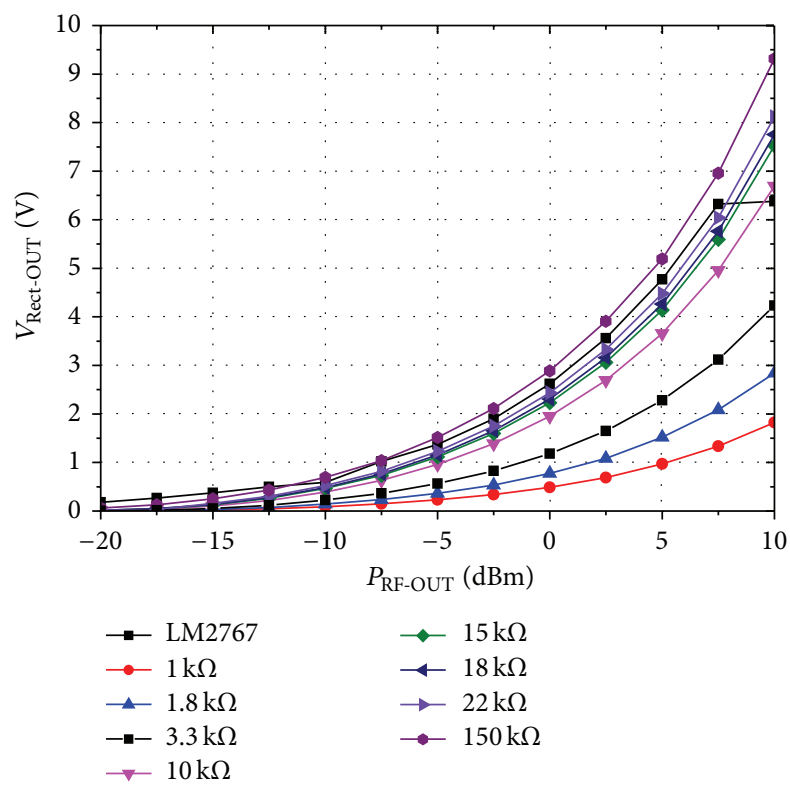

FIGURE 13: $V_{\text {Rect-OUT }}$ as function of $P_{\text {RF-OUT }}$ for different resistive loads are compared with the one obtained when the rectifier is connected with the boost circuit (see Figure 9).

In Figure 13, the curves obtained for $V_{\text {Rect-OUT }}$ as a function of $P_{\mathrm{RF}-\mathrm{OUT}}$ for different resistive loads are compared with the one obtained when the rectifier is connected with the boost circuit (see Figure 9). This comparison demonstrates the validity of the initial assumptions, and in particular that rectifier sees the boost circuit as a resistive load of kilohms order of magnitude.

3.3. Battery Charger. The battery charger, whose circuit diagram is given in Figure 9(b), was implemented by using the LTC4071 chip by Linear Technologies [30]. The main feature of the LTC4071 is the charging of low capacity Li-ion or thinfilm batteries from very low current. For a correct operation, the LTC4071 requires just two external lumped components, namely, a resistor and a capacitor (see Figure 9(b)). The aim of the capacitor is to decouple the VCC and the GND pins; according to specifications (which recommend using a capacitor higher than $0.1 \mu \mathrm{F}$ ), a $10 \mu \mathrm{F}$ capacitor was used for $C_{8}$. As for $R_{\mathrm{IN}}$, a resistor of $180 \Omega$ was used.

The VCC pin is connected to the source, by means of the series resistor $R_{\mathrm{IN}}$; in presence of a system load, this must also be connected to VCC pin, as shown in Figure 9. The battery is connected to the BAT pin.

The LTC4071 chip has two different operation modes: the "recharge mode" and the "user mode." The recharge mode allows recharging a battery connected between the GND and the BAT pin; in this case, the power source is on and the power provided by the source is used to recharge the battery. In the user mode, the power source is off and the system load connected to the VCC pin is powered through the battery. In the user mode, the DC voltage on the VCC pin is controlled by the ADJ pin; in this work, the pin ADJ is floating, which corresponds to a voltage of $4.1 \mathrm{~V}$ on the VCC pin.
The LTC4071 implements also a battery protection from both overcharge and overdischarge. In particular, to protect the battery, the voltage on the BAT pin $\left(V_{\mathrm{BAT}}\right)$ is monitored and the power flow from the battery to the system load is interrupted when $V_{\mathrm{BAT}}$ is lower than a threshold voltage $V_{\mathrm{LBD}}$. In turn, $V_{\mathrm{LBD}}$ is set through an appropriate connection of the pin LBSEL. More specifically, connecting the LBSEL pin to ground corresponds to set $V_{\mathrm{LDB}}$ at $3.2 \mathrm{~V}$, while connecting the LBSEL pin to VCC corresponds to set $V_{\mathrm{LDB}}$ at $2.7 \mathrm{~V}$. As for the protection from overcharge, when a source is present, the battery charges until the battery voltage rises above the low battery connect voltage $\left(V_{\mathrm{LBC}}\right)$. Also $V_{\mathrm{LBC}}$ depends on the state of the LBSEL pin, but its value varies with respect to other parameters, such as charge current (for more details, refer to [30]). In this work, the LBSEL pin was connected to ground.

As for the battery, a THINERGY MEC201 by Infinite Power Solutions (IPS), having a battery capacity of $0.7 \mathrm{mAh}$, was chosen. In the following section, the experimental results for the charging time are presented and discussed.

\section{Wireless Charger for Thin-Film Batteries: Experimental Results}

The performance of the proposed battery charger consisting of the WREL illustrated in Figure 6 connected to the PMU of Figure 10 was experimentally investigated. In more detail, experimental tests were performed in order to evaluate both the total efficiency of the wireless charger $\left(\eta_{\mathrm{TOT}}\right)$ and the time necessary to recharge the THINERGY MEC201 battery $\left(t_{\mathrm{CHG}}\right)$.

As for the total efficiency, $\eta_{\mathrm{TOT}}$, we refer to the following definition:

$$
\eta_{\mathrm{TOT}}=\frac{P_{\mathrm{DC}-\mathrm{OUT}}}{P_{\mathrm{RF}-\mathrm{IN}}}=\frac{V_{\mathrm{BAT}} \cdot I_{\mathrm{CHG}}}{P_{\mathrm{RF}-\mathrm{IN}}},
$$

where $V_{\mathrm{BAT}}$ and $I_{\mathrm{CHG}}$ are the charge voltage and the charge current, respectively.

Referring to Figure 1, experimental tests were performed through the VSG R\&S FSW26 to generate the RF input power $\left(P_{\text {RF-IN }}\right)$. The input port of the WREL, configured as illustrated in Figure 6(b), was connected to the VSG by means of a flexible coaxial cable, while the output port was connected to the PMU by means of a $50 \Omega$ SMA connector. As input RF signal, a $434 \mathrm{MHz}$ sinusoidal signal was used.

Figures 14(a) and 14(b) show the results for $\eta_{\mathrm{TOT}}$ and $t_{\mathrm{CHG}}$, respectively. From Figure 14(a), it can be seen that the proposed wireless charger exhibits a maximum $\eta_{\text {TOT }}$ of about 29.7\% for an RF input power of $7.5 \mathrm{dBm}$.

As for the charging time, experimental tests demonstrated that values of $P_{\mathrm{RF}-\mathrm{IN}}$ higher than $1.5 \mathrm{dBm}$ are necessary to recharge the THINERGY MEC201 battery.

Figure 14(b) shows results obtained for the charging time (i.e., $t_{\mathrm{CHG}}$ ) of the low capacity thin-film battery THINERGY MEC201 as a function of the power delivered to the primary resonator. It can be seen that, for $P_{\mathrm{RF}-\mathrm{IN}}$ higher than $6 \mathrm{dBm}$, the time necessary to recharge the THINERGY MEC201 battery is shorter than 50 minutes. 


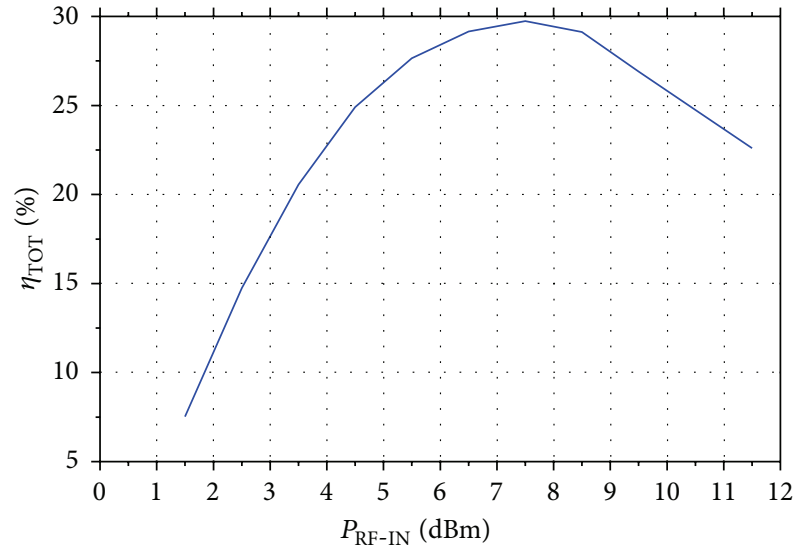

(a)

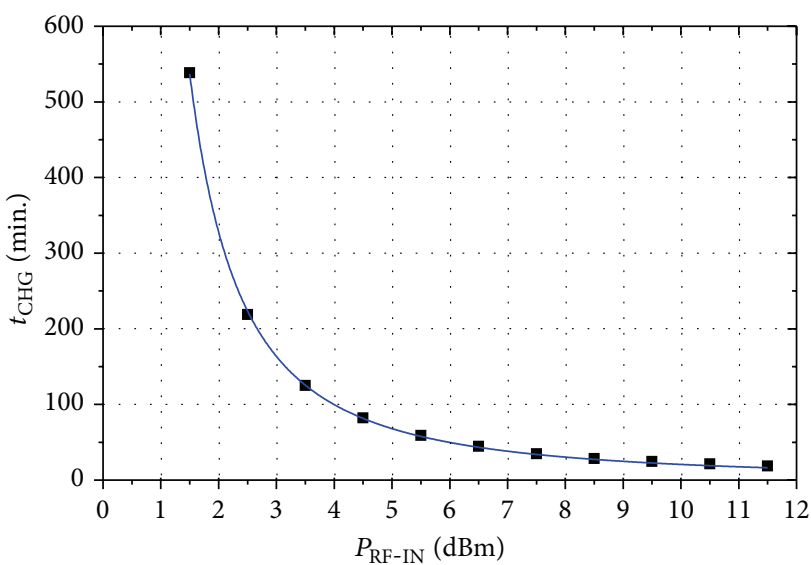

(b)

FIGURE 14: Experimental results obtained for the proposed wireless battery charger: (a) total efficiency as function of the input power $\left(P_{\text {RF-IN }}\right)$ at the primary resonator (see Figure 1); (b) charging time necessary to recharge the battery THINERGY MEC201 as function of input power $\left(P_{\mathrm{RF}-\mathrm{IN}}\right)$ at the primary resonator (see Figure 1$)$.

\section{Conclusion}

In this work, a wireless charger for low capacity thin-film batteries was presented. The proposed device exploits a nonradiative wireless resonant energy link for power transmission. A prototype operating in the ISM band centered at $434 \mathrm{MHz}$ was presented and discussed. The power link was fabricated by using an adhesive conductive nonwoven fabric on a leather substrate, thus resulting in a device suitable to be embedded in wearable accessories (such as handbags). From experimental data, it was demonstrated that the fabricated power link exhibits a maximum of the RF-to-RF power transfer efficiency of about $69.3 \%$.

As for the battery charger, a total efficiency of approximately $29.7 \%$ was demonstrated.

It was also shown that, by providing an input power higher than $6 \mathrm{dBm}$, a time charging shorter than 50 minutes is required for recharging a thin-film battery THINERGY MEC201.

Overall, the obtained results demonstrate the feasibility of using the proposed wearable WPT link for implementing a wireless charger.

\section{Competing Interests}

The authors declare that there is no conflict of interests regarding the publication of this paper.

\section{References}

[1] M. A. R. Osman, M. K. A. Rahim, N. A. Samsuri, H. A. M. Salim, and M. F. Ali, "Embroidered fully textile wearable antenna for medical monitoring applications," Progress in Electromagnetics Research, vol. 117, pp. 321-337, 2011.

[2] Y. Li, R. Vyas, A. Rida, J. Pan, and M. M. Tentzeris, "Wearable RFID-enabled sensor nodes for biomedical applications," in Proceedings of the 58th Electronic Components and Technology
Conference (ECTC '08), pp. 2156-2159, IEEE, Lake Buena Vista, Fla, USA, May 2008.

[3] C. Hertleer, H. Rogier, and L. Van Langenhove, "A textile antenna for protective clothing," in Proceedings of the IET Seminar on Antennas and Propagation for Body-Centric Wireless Communications, pp. 44-46, April 2007.

[4] L. Vallozzi, P. Van Torre, C. Hertleer, H. Rogier, M. Moeneclaey, and J. Verhaevert, "Wireless communication for firefighters using dual-polarized textile antennas integrated in their garment," IEEE Transactions on Antennas and Propagation, vol. 58, no. 4, pp. 1357-1368, 2010.

[5] Z. Chun-Qing, W. Jun-Hong, and H. Yu-Nan, "Coupled planar dipole UWB antenna design for wearable computer," in Proceedings of the International Conference on Microwave and Millimeter Wave Technology (ICMMT '07), pp. 1-4, April 2007.

[6] W. Thompson, R. Cepeda, G. Hilton, M. A. Beach, and S. Armour, "An improved antenna mounting for ultra-wideband on-body communications and channel characterization," IEEE Transactions on Microwave Theory and Techniques, vol. 59, no. 4, pp. 1102-1108, 2011.

[7] B. Sanz-Izquierdo, J. A. Miller, J. C. Batchelor, and M. I. Sobhy, "Dual-band wearable metallic button antennas and transmission in body area networks," IET Microwaves, Antennas and Propagation, vol. 4, no. 2, pp. 182-190, 2010.

[8] H. Marques and B. V. Borges, "Contactless battery charger with high relative separation distance and improved efficiency," in Proceedings of the 33rd International Telecommunications Energy Conference (INTELEC '11), pp. 1-8, Amsterdam, The Netherlands, October 2011.

[9] K.-C. Wan, Q. Xue, X. Liu, and S. Y. Hui, "Passive radiofrequency repeater for enhancing signal reception and transmission in a wireless charging platform," IEEE Transactions on Industrial Electronics, vol. 61, no. 4, pp. 1750-1757, 2014.

[10] A. N. Laskovski, M. R. Yuce, and T. Dissanayake, "Stacked spirals for biosensor telemetry," IEEE Sensors Journal, vol. 11, no. 6, pp. 1484-1490, 2011.

[11] K. Jung, Y.-H. Kim, E. J. Choi, H. J. Kim, and Y.-J. Kim, “Wireless power transmission for implantable devices using inductive 
component of closed magnetic circuit," Electronics Letters, vol. 45, no. 1, pp. 21-22, 2009.

[12] G. Monti, L. Tarricone, M. Dionigi, and M. Mongiardo, "Magnetically coupled resonant wireless power transmission: an artificial transmission line approach," in Proceedings of the 42nd European Microwave Conference (EuMC '12), pp. 233-236, IEEE, Amsterdam, The Netherlands, October-November 2012.

[13] A. Costanzo, M. Dionigi, D. Masotti et al., "Electromagnetic energy harvesting and wireless power transmission: a unified approach," Proceedings of the IEEE, vol. 102, no. 11, pp. 1692-1711, 2014.

[14] S. Rivière, A. Douyère, F. Alicalapa, and J. D. Lan Sun Luk, "Study of complete WPT system for WSN applications at low power level," Electronics Letters, vol. 46, no. 8, pp. 597-598, 2010.

[15] G. Monti, L. Corchia, and L. Tarricone, "UHF wearable rectenna on textile materials," IEEE Transactions on Antennas and Propagation, vol. 61, no. 7, pp. 3869-3873, 2013.

[16] D. Masotti, A. Costanzo, and S. Adami, "Design and realization of a wearable multi-frequency RF energy harvesting system," in Proceedings of the 5th European Conference on Antennas and Propagation, pp. 517-520, April 2011.

[17] G. Monti, L. Corchia, and L. Tarricone, "Textile logo antennas," in Proceedings of the 14th Mediterranean Microwave Symposium (MMS '14), pp. 1-5, Marrakech, Morocco, December 2014.

[18] G. Monti and F. Congedo, "UHF rectenna using a bowtie antenna," Progress in Electromagnetics Research C, vol. 26, pp. 181-192, 2012.

[19] G. Monti, L. Corchia, and L. Tarricone, "A microstrip antenna with a reconfigurable pattern for RFID applications," Progress In Electromagnetics Research B, no. 45, pp. 101-116, 2012.

[20] G. Monti, L. Corchia, and L. Tarricone, "Planar bowtie antenna with a reconfigurable radiation pattern," Progress in Electromagnetics Research C, vol. 28, pp. 61-70, 2012.

[21] F. Congedo, G. Monti, L. Tarricone, and M. Cannarile, "Broadband bowtie antenna for RF energy scavenging applications," in Proceedings of the 5th European Conference on Antennas and Propagation (EUCAP '11), pp. 335-337, Rome, Italy, April 2011.

[22] G. Monti, L. Corchia, and L. Tarricone, "A wearable wireless energy link," in Proceedings of the 45th European Microwave Conference (EuMC '15), pp. 143-146, Paris, France, September 2015.

[23] M. Dionigi, A. Costanzo, and M. Mongiardo, "Network methods for analysis and design of resonant wireless power transfer systems," in Wireless Power Transfer-Principles and Engineering Explorations, K. Y. Kim, Ed., InTech, 2012, http:// www.intechopen.com/books/wireless-power-transfer-principles-and-engineering-explorations/networks-methods-for-theanalysis-and-design-of-wireless-power-transfer-systems.

[24] ADFORS Saint-Gobain, http://www.sg-adfors.com/Technologies/Fabrics/Glassmat.

[25] G. Monti, L. Corchia, and L. Tarricone, "Fabrication techniques for wearable antennas," in Proceedings of the European Radar Conference (EuRAD '13), Nuremberg, Germany, October 2013.

[26] G. Monti, L. Corchia, and L. Tarricone, "Logo antenna on textile materials," in Proceedings of the European Microwave Conference (EuMC '14), pp. 516-519, Rome, Italy, October 2014.

[27] http://www.awrcorp.com/products.

[28] "HSMS-282x: Surface Mount RF Schottky Barrier Diodes," http://www.avagotech.com/docs/AV02-1320EN.

[29] http://www.ti.com/general/docs/lit/getliterature.tsp?genericPartNumber $=\operatorname{lm} 2767 \&$ fileType $=$ pdf.

[30] http://www.linear.com/product/LTC4071. 


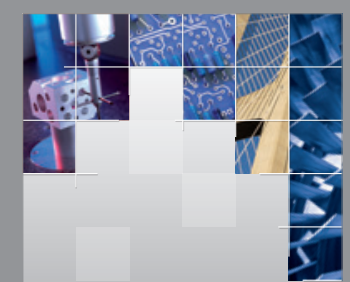

\section{Enfincering}
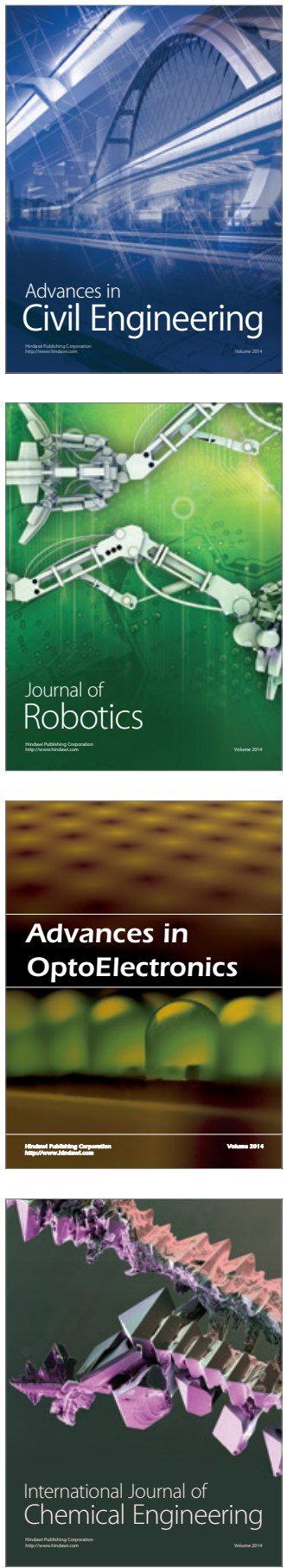

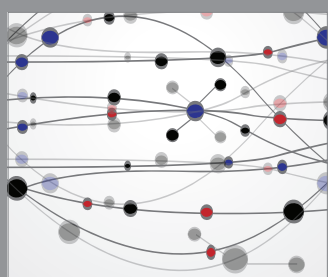

The Scientific World Journal

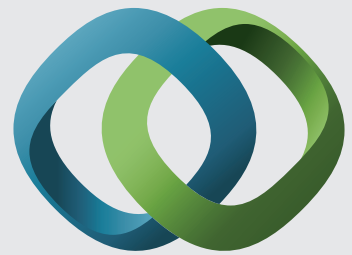

\section{Hindawi}

Submit your manuscripts at

http://www.hindawi.com
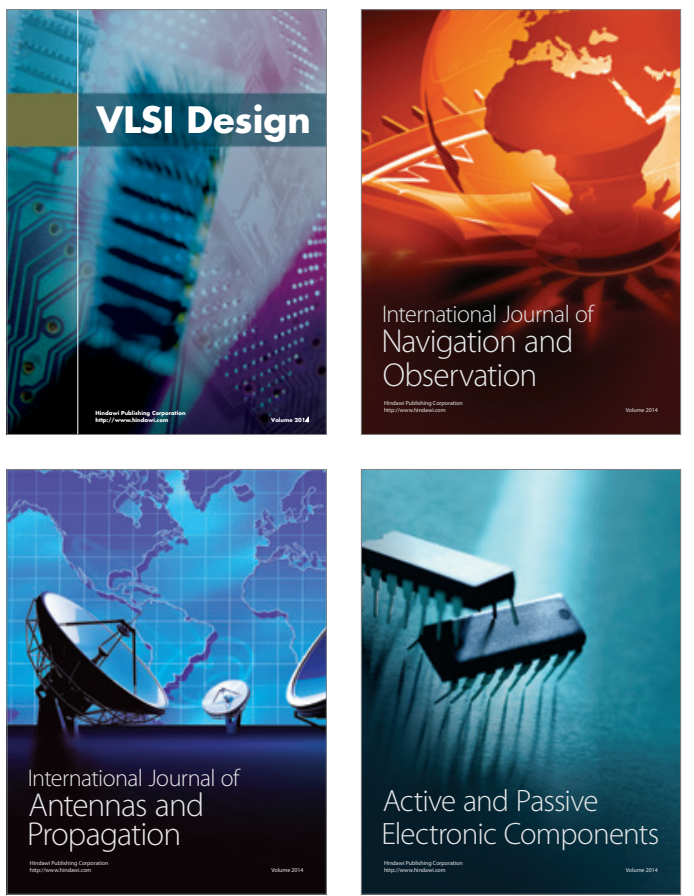
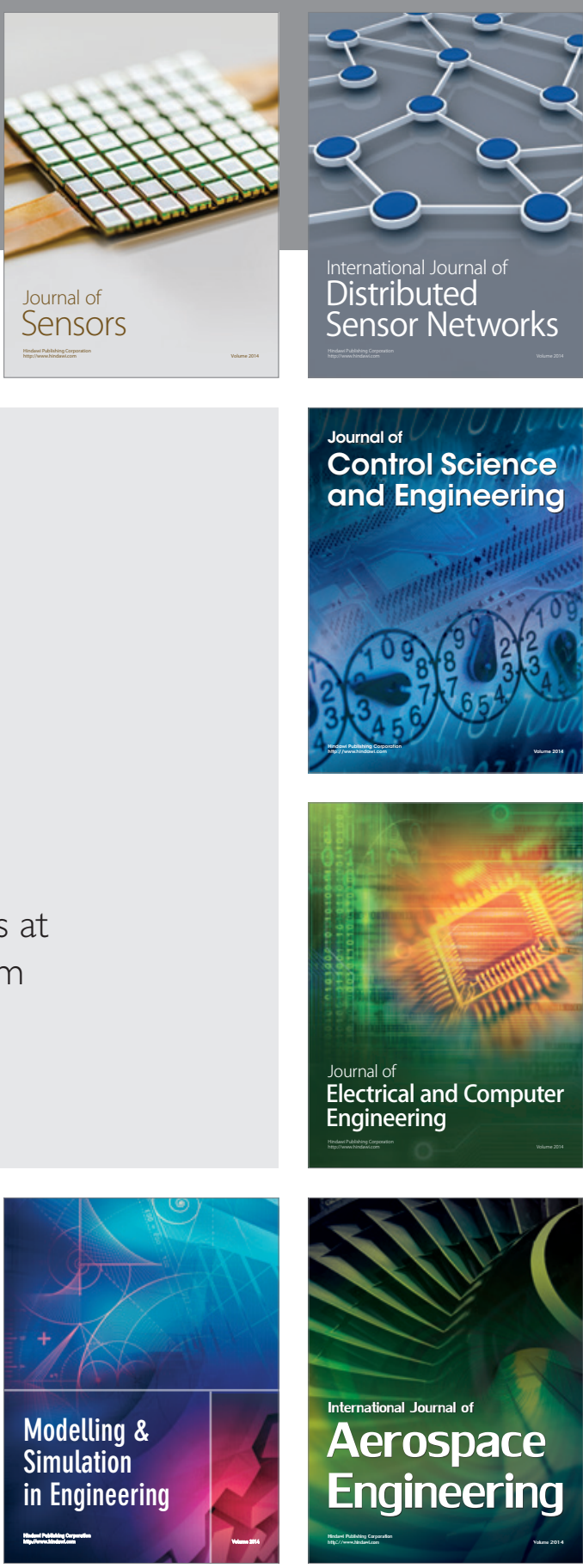

International Journal of

Distributed

Sensor Networks

Journal of

Control Science

and Engineering
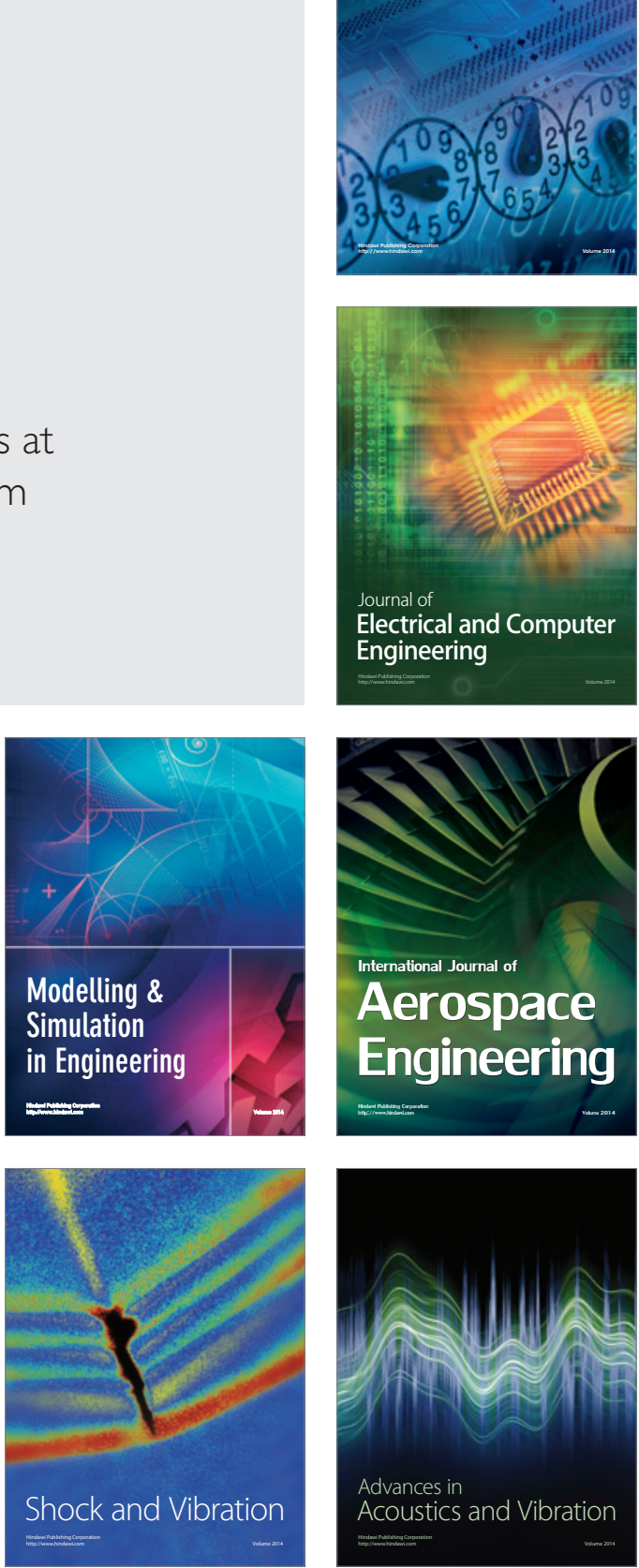